\title{
Rapid generation of broad T-cell immunity in humans after a single injection of mature dendritic cells
}

\author{
Madhav V. Dhodapkar, ${ }^{1}$ Ralph M. Steinman, ${ }^{1}$ Mark Sapp,${ }^{1}$ Hema Desai, ${ }^{1}$ \\ Coraleen Fossella, ${ }^{2}$ Joseph Krasovsky, ${ }^{1}$ Sean M. Donahoe, ${ }^{3}$ P. Rod Dunbar, ${ }^{4}$ \\ Vincenzo Cerundolo, ${ }^{4}$ Douglas F. Nixon, ${ }^{3}$ and Nina Bhardwaj ${ }^{1}$ \\ ${ }^{1}$ Laboratory of Immunology and Cellular Physiology, \\ ${ }^{2}$ General Clinical Research Center, and \\ ${ }^{3}$ Aaron Diamond AIDS Research Center, Rockefeller University, New York, New York 10021, USA \\ ${ }^{4}$ Molecular Immunology Group, Institute of Molecular Medicine, John Radcliffe Hospital, University of Oxford, \\ Oxford OX3 9OU, United Kingdom
}

Address correspondence to: Madhav Dhodapkar, Laboratory of Immunology and Cellular Physiology, Rockefeller University,

1230 York Avenue, Box 176, New York, New York 10021, USA. Phone: (212) 327-7597; Fax: (212) 327-8875;

E-mail: dhodapm@rockvax.rockefeller.edu.

Received for publication March 25, 1999, and accepted in revised form June 10, 1999.

\begin{abstract}
Dendritic cells (DCs) are potent antigen-presenting cells that initiate protective T-cell immunity in mice. To study the immunogenicity of DCs in humans, we injected 9 healthy subjects subcutaneously with a control injection of autologous monocyte-derived, mature DCs, followed 4-6 weeks later by DCs pulsed with keyhole limpet hemocyanin $(\mathrm{KLH}), \mathrm{HLA}-\mathrm{A}^{*} 0201$-positive restricted influenza matrix peptide (MP), and tetanus toxoid (TT). Four more subjects received these antigens without DCs. Injection of unpulsed DCs, or antigens alone, failed to immunize. Priming of $\mathrm{CD}^{+} \mathrm{T}$ cells to KLH was observed in all 9 subjects injected with KLH-pulsed DCs, and boosting of TT-specific Tcell immunity was seen in 5 of 6 subjects injected with TT-pulsed DCs. Injection of antigen-pulsed DCs led to a severalfold increase in freshly isolated MP-specific, IFN- $\gamma$-secreting $\mathrm{CD}^{+} \mathrm{T}$ cells in all 6 HLA-A*0201-positive subjects, as early as 7 days after injection. When T cells were boosted in culture, there was an increase in MHC tetramer-binding cells and cytotoxic T cells after DC vaccination. These data provide the first controlled evidence of the immunogenicity of DCs in humans, and demonstrate that a single injection of mature DCs rapidly expands T-cell immunity.
\end{abstract}

J. Clin. Invest. 104:173-180 (1999).

\section{Introduction}

Most conventional vaccines are composed of inactivated pathogens or their subunits, and primarily elicit humoral immunity. In contrast, it has proved difficult to induce combined $\mathrm{CD}^{+}$and $\mathrm{CD}^{+} \mathrm{T}$-cell immunity in humans (1). Dendritic cells (DCs) are antigen-presenting cells (APCs) specialized to initiate T-cell immunity, including cytotoxic T lymphocytes (CTLs) that kill virusinfected or malignant targets (2). DCs normally reside in tissues in an immature form, where they are specialized to capture antigen. After antigen capture, and in response to inflammatory stimuli, DCs switch to a $\mathrm{T}$ cell-stimulatory mode by a process termed maturation and migrate to lymph nodes to initiate immunity (2). Maturation of DCs is associated with upregulation of costimulatory molecules, severalfold enhancement of their APC function, and expression of chemokine receptors that promote migration to nodal T-cell areas (3).

In several animal studies, immunization with antigenbearing DCs efficiently primes both $\mathrm{CD}^{+}$helper and $\mathrm{CD}^{+}$cytotoxic T-cell immunity. This leads to protective immunity against tumors and infectious agents (4-7). There have been preliminary reports of using immature DCs in patients with malignancies (8-12). These studies mostly emphasized the issues of safety and suggested potential antitumor efficacy. However, it is difficult to assess the immunogenicity of DCs in these studies because the $\mathrm{CD}^{+} \mathrm{T}$-cell immunity was not quantified and because in some of the studies, the patients often also received soluble antigens, i.e., keyhole limpet hemocyanin $(\mathrm{KLH})$ or idiotype protein in addition to DCs (8). Evaluation of T-cell immunity was largely based on delayed-type hypersensitivity (DTH) reactions or in vitro proliferative responses after multiple DC doses; unpulsed DC controls were not tested. Additionally, in some studies, DCs were grown in the presence of FCS, which is known to induce syngeneic T-cell responses in culture and could have produced nonspecific immunomodulatory effects in vivo (10, 12). The biology of DCs is sufficiently intricate that their use as adjuvants will require quantitative T-cell assays to identify optimal immunization conditions (13).

We have developed a method to reproducibly generate mature DCs from blood monocyte precursors (14). We chose these mature DCs for active immunization, as they are more potent APCs in vitro and do not revert to macrophages after withdrawal of cytokines, as in the case of immature DCs used in other studies (14). We targeted healthy subjects to enable us to study DC biology in vivo in humans without the confounding variables of tumorassociated immunodeficiency or comorbidity. Here we use several parameters of antigen-specific T-cell function, including newer quantitative assays $(15,16)$ to show that 
Table 1

Patient characteristics

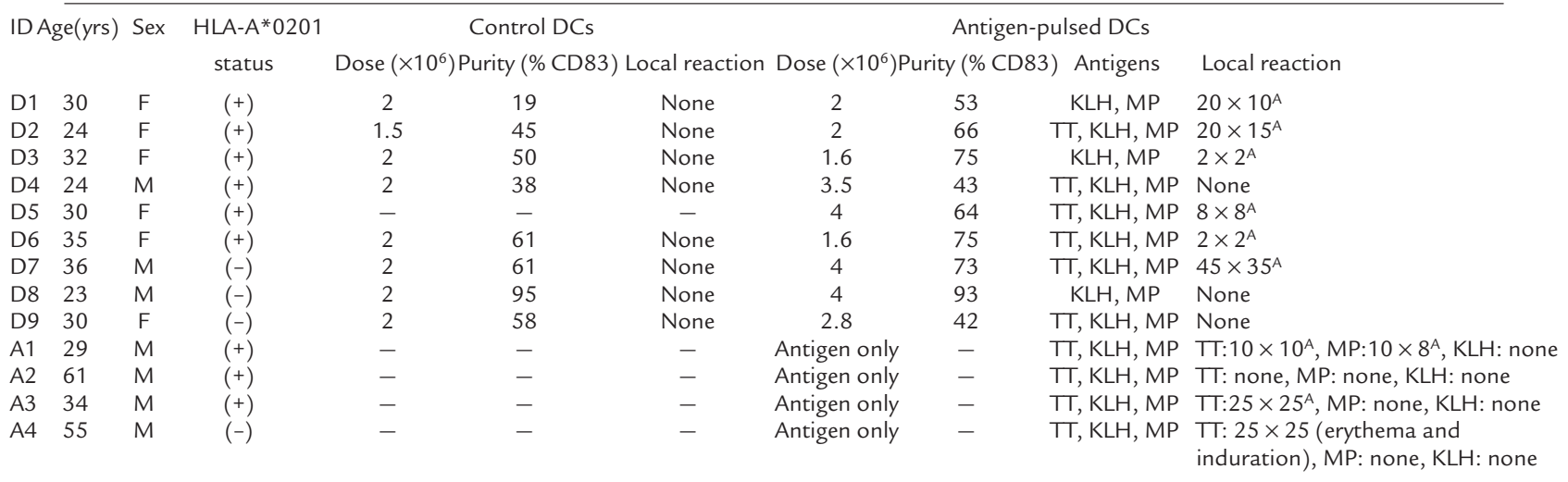

AErythema (mm). F, female; M, male.

antigen-pulsed mature DCs rapidly generate broad T-cell immunity in humans. The T-cell responses are evident in both $\mathrm{CD}^{+}$and $\mathrm{CD}^{+} \mathrm{T}$-cell compartments.

\section{Methods}

Study design. The schema for DC injection is shown in Figure 1. As additional controls, 4 subjects received subcutaneous injections of antigens alone without adjuvants.

Human subjects. Normal healthy adult subjects were recruited through advertisement. Eligibility criteria were as follows: age, 18-65 years; no history of chronic infection (e.g., hepatitis, tuberculosis) or malignancy; and acceptable hepatic (serum transaminases $<3$ upper limit normal), renal (serum creatinine $<1.5$ upper limit normal), and hematologic function (hematocrit $>30 \%$, granulocytes $>1,500 / \mu \mathrm{L}$, and platelets $>100,000 / \mu \mathrm{L})$. Individuals with psychiatric disorders, active substance abuse, allergy to gentamicin (used in the culture medium), pregnancy, or autoimmune disease (including positive rheumatoid factor or serum antinuclear antibody [ANA]) were excluded. The study was approved by the Food and Drug Administration. All subjects signed an informed consent in accordance with the Rockefeller University Institutional Review Board guidelines.

Baseline studies. All study participants were typed for HLA-A*0201 and were initially followed up for a 2- to 12 -month period, during which $2-4$ baseline measure-

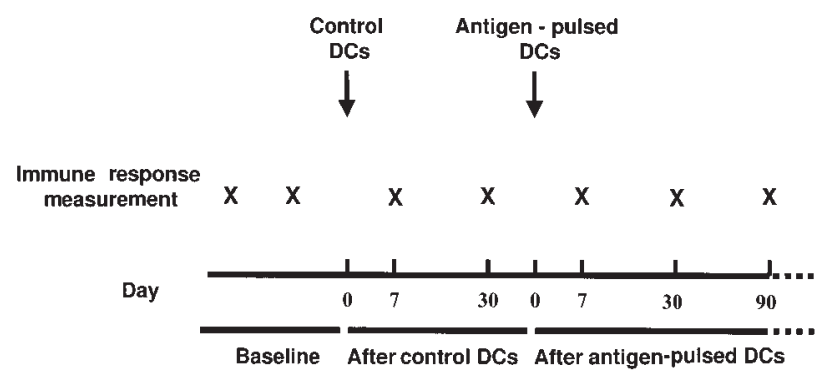

Figure 1

Study schema for DC injection. Four additional controls received antigen only. ments of immune response were made. Laboratory tests at baseline included complete blood count, chemistry profile, hepatitis B and C serology, HIV serology, rheumatoid factor, ANA, sedimentation rate, urinalysis, chest $\mathrm{x}$-ray, pregnancy test, and influenza serology. An anergy panel consisting of candida, mumps, and tetanus was placed at baseline in all but 2 subjects (D4 and D7).

\section{Measurement of immune responses}

Antigen-specific proliferation. PBMCs at 2 dose levels $(3 \times$ $10^{4}$ and $1 \times 10^{5}$ cells per well) were cultured in triplicate wells in the absence or presence of graded doses of antigens. The antigen and doses were as follows: tetanus toxoid (TT) at 3-3,000 ng/mL, KLH at $1-10 \mu \mathrm{g} / \mathrm{mL}$, and as a positive control, $0.001-1.0 \mathrm{ng} / \mathrm{mL}$ staphylococcal enterotoxin A (SEA). Assays were pulsed with $\left[{ }^{3} \mathrm{H}\right]$ thymidine after 3 (SEA) or 5 (TT and KLH) days of culture and were harvested 8 hours later. For some experiments, PBMCs were depleted of $\mathrm{CD}^{+}{ }^{+}$or $\mathrm{CD}^{+} \mathrm{T}$ cells by panning before use in the proliferation and enzyme-linked immunospot (ELISPOT) assays.

ELISPOT assay for IFN- $\gamma$ release from single antigen-specific T cells. As described elsewhere $(16,17)$, graded doses of PBMCs $\left(25 \times 10^{3}\right.$ to $2 \times 10^{5}$ cells per well) were added to plates precoated with $10 \mu \mathrm{g} / \mathrm{mL}$ of a primary anti-IFN$\gamma \mathrm{mAb}$ (Mabtech, Stockholm, Sweden) in the presence or absence of antigens, to stimulate IFN- $\gamma$ secretion. The plates were incubated overnight $(12-18$ hours $)$ at $37^{\circ} \mathrm{C}$, and then the wells were incubated with biotin-conjugated anti-IFN- $\gamma$ antibody $(1 \mu \mathrm{g} / \mathrm{mL}$; Mabtech). After staining with Vectastain Elite kit (Vector Laboratories, Burlingame, California, USA), colored spots indicated the IFN- $\boldsymbol{\gamma}$-secreting cells. Antigens were as follows: 10 $\mu \mathrm{M}$ HLA-A*0201 restricted influenza matrix peptide (MP) (GILGFVFTL) (18) and $1 \mu \mathrm{g} / \mathrm{mL}$ HLA-A*0201 restricted HIV gag peptide (SLYNTVATL) (as control). For the detection of influenza-specific responses, PBMCs were infected with live influenza virus $(\mathrm{moi}=2)$. Responses were counted as positive if a minimum of 10 spot-forming cells (SFCs) per $2 \times 10^{5}$ cells were detected after the control had been subtracted, and if the numbers of spots were at least twice those in the negative control wells. All assays were performed on fresh 
PBMCs. Where feasible, for some subjects, aliquots of PBMCs from before and after immunization were also thawed and assayed together.

CTL assays. MP-specific killer cells were quantified after T-cell stimulation $\left(2 \times 10^{5} \mathrm{~T}\right.$ cells per well $)$ with antigenpulsed DCs (influenza MP [1 $\mu \mathrm{M}]$, live or heat-inactivated influenza virus [moi $=2]$ ) at a DC/T ratio of 1:30 for 7 days $(19,20)$. CTL activity was measured in a standard 5hour ${ }^{51} \mathrm{Cr}$-release assay at an effector/target $(\mathrm{E} / \mathrm{T})$ ratio of 20:1. For HLA-A*0201-positive patients, targets were T2 cells pulsed with $1 \mu \mathrm{M} \mathrm{MP}$, or unpulsed T2 cells as controls. In HLA-A*0201-negative patients, autologous B lymphoblastoid cell lines infected with live influenza (moi $=10)$ or vaccinia vectors $(\mathrm{moi}=5)$ carrying influenza matrix protein or thymidine kinase gene (as control; a gift of B. Moss, National Institutes of Health, Bethesda, Maryland, USA) served as targets.

Binding to HLA class I tetramer complexes. Soluble influenza MP-HLA-A*0201 tetramers were prepared, and binding to tetramers was analyzed by $\mathrm{FACS}^{\circledR}$ analysis (21). Frozen aliquots of PBMCs from before and after immunization were thawed together in a blinded fashion. The PBMCs were cultured in vitro with freshly generated autologous DCs pulsed with MP (versus no peptide as control) at a DC/T ratio of 1:30 for 7 days before staining and analysis.

Generation of DCs. DCs were generated from blood monocyte precursors as described (22). Briefly, mononuclear cells were plated in 6 -well culture plates at $8 \times 10^{6}$ cells per well and allowed to adhere to plastic for 2 hours. Plastic adherent cells were cultured in RPMI-1640 (BioWhittaker, Walkersville, Maryland, USA) supplemented with $1 \%$ autologous plasma, $20 \mu \mathrm{g} / \mathrm{mL}$ gentamicin (clinical grade; Fujisawa USA, Deerfield, Illinois, USA), 800 U/mL GMP grade IL-4 (Cell Genix, Freiburg, Germany), and $100 \mathrm{IU} / \mathrm{mL}$ clinical grade GM-CSF (Immunex, Seattle, Washington, USA) for 7 days. Cultures were supplemented with cytokines on days 2 , 4 , and 6 . On day 7, cells were transferred to new plates and cultured in the presence of $50 \%$ (vol $/ \mathrm{vol})$ monocyte-conditioned medium (MCM) for 2 additional days. Using this methodology, $1.5 \times 10^{6}$ to $4 \times 10^{6}$ mature DCs expressing high levels of CD86, CD83, and HLA-DR, and lacking other lineage markers (e.g., CD14), were reproducibly generated from $80 \mathrm{~mL}$ of peripheral blood. MCM was prepared by adhering $50 \times 10^{6} \mathrm{PBMCs}$ in $7 \mathrm{~mL}$ of $1 \%$ autologous plasma to plates precoated with $100 \mu \mathrm{g} / \mathrm{mL}$ human IgG (Bayer Corp., Elkhart, Indiana, USA) for 90 minutes. The PBMCs were harvested after overnight culture of adherent cells and were filtered and frozen at $-20^{\circ} \mathrm{C}$ before use. We have shown previously that mature DCs generated using this methodology are potent stimulators in mixed lymphocyte reaction at $\mathrm{T} / \mathrm{DC}$ ratios of 900:1 (22).

$D C$ injection. The first injection was a control injection in which no antigen was added. This was followed 4-6 weeks later by the injection of antigen-pulsed DCs. Antigens were added to DCs overnight 1 day before injection. The antigens were as follows: $10 \mu \mathrm{g} / \mathrm{mL} \mathrm{KLH}$ (depyrogenated, LPS free; Calbiochem-Novabiochem Corp., San Diego, California, USA), $10 \mu \mathrm{g} / \mathrm{mL}$ TT (Statens Serum Institute, Copenhagen, Denmark), and $1 \mu \mathrm{M}$ HLA$A^{*} 0201$ restricted influenza MP (manufactured under
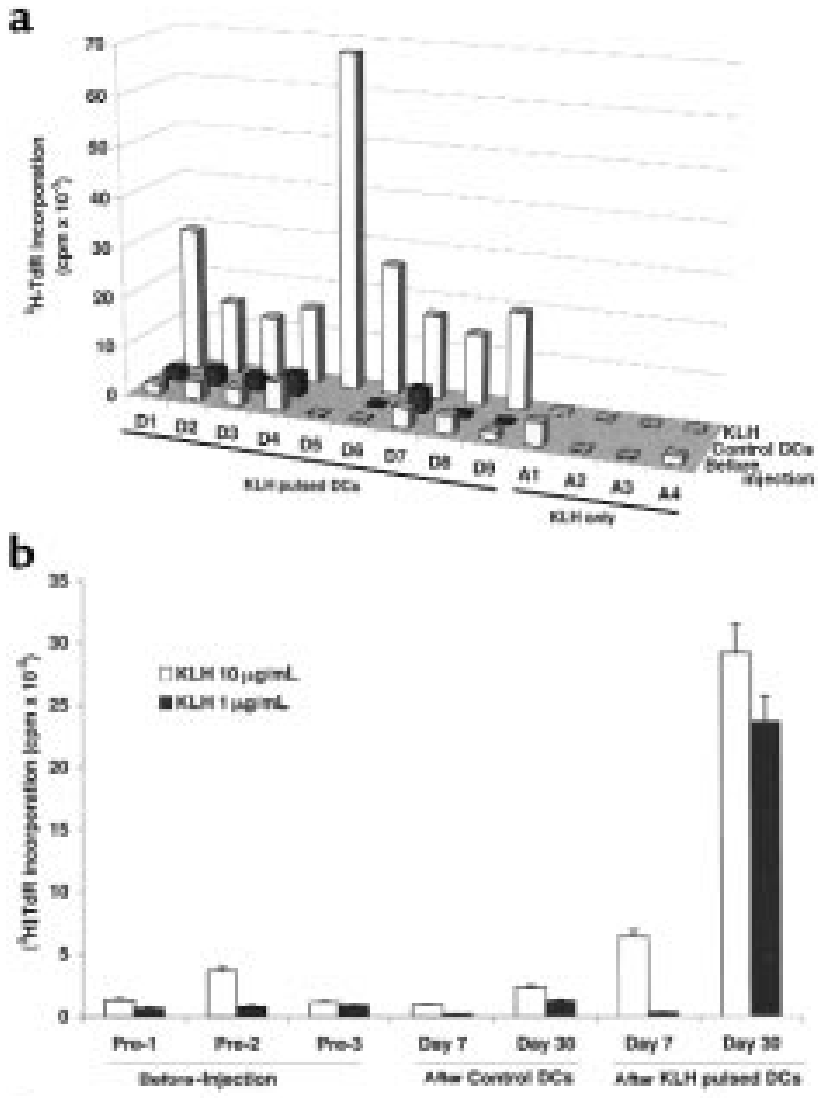

c

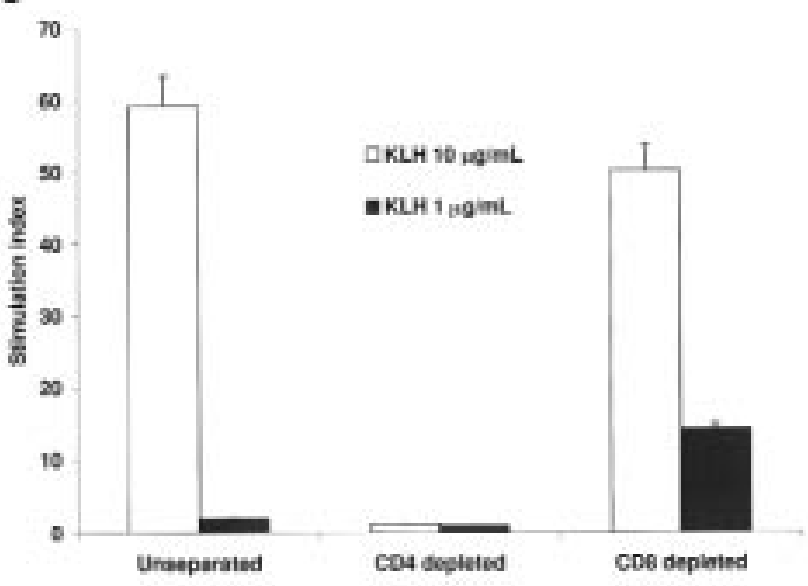

Figure 2

(a) Priming to $\mathrm{KLH}$ after $\mathrm{KLH}$-pulsed DCs. KLH-specific proliferation at baseline and at 30 days after control DC injection and KLH-pulsed DC injection are shown (except in D5, who demonstrated priming only at day 60). For A1-A4, data shown are for baseline and 30 days after antigen injection. For each measurement shown, $10^{5}$ PBMCs were incubated with $10 \mu \mathrm{g} / \mathrm{mL} \mathrm{KLH}$ for 5 days. Results are expressed as $\left[{ }^{3} \mathrm{H}\right] \mathrm{TdR}$ incorporation in counts per minute. SEM for all measurements was $<20 \%$. (b) Kinetics of KLH priming as determined by antigen-specific proliferation in a representative subject (D1). For each assay, $3 \times 10^{4}$ or $1 \times 10^{5}$ PBMCs per well were incubated with 1 or $10 \mu \mathrm{g} / \mathrm{mL} \mathrm{KLH}$ for 5 days. Results, as shown for $10^{5}$ PBMCs per well, are expressed as $\left[{ }^{3} \mathrm{H}\right] \mathrm{TdR}$ incorporation in counts per minute. (c) CD4 nature of the $\mathrm{KLH}$ proliferative response. $\mathrm{KLH}$-specific proliferation in unseparated PBMCs was compared with that after $\mathrm{CD} 4^{+}$ and $C D 8^{+} T$-cell depletion by panning. 


\section{Figure 3}

Boosting of TT-specific immunity after TT-pulsed DCs. TT-specific proliferation was measured at baseline and at 30 days after control DC injection and TT-pulsed DC injection. In D1, D3, and D8, TT was omitted from the antigen-pulsed DCs because of strong baseline DTH responses. For A1-A4, data shown are for baseline and 30 days after antigen-only injection. For each measurement shown, $10^{5}$ PBMCs were incubated with $3 \mu \mathrm{g} / \mathrm{mL}$ TT for 5 days. Results are expressed as stimulation index. SEM for all measurements was $<20 \%$.

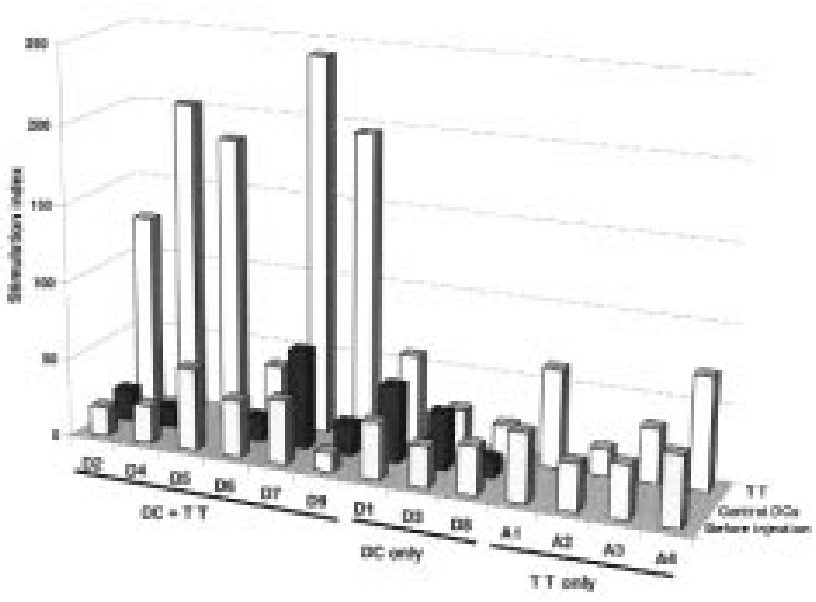

rent acute influenza infection during the study.

Statistical analysis. For analysis of the immune response, values before and after immunization were compared by paired $t$ test after logarithmic transformation of the data. Generation of a relevant immune response was defined before the study as $\geq 2$-fold increase in antigen-specific proliferation for KLH and TT, or the number of IFN$\gamma$-producing cells in the ELISPOT assay. Significance was set at $P<0.05$. cutaneous injection in 2 adjacent sites on the upper inner arm, $\sim 4$ inches from the axilla. All injected DC preparations tested negative for bacterial and fungal contamination. All subjects were observed overnight at the Rockefeller University Hospital.

Antigen-only controls. In 4 control subjects, the study antigens (TT, KLH, and MP) were injected without DCs or other adjuvant, at $10 \mu \mathrm{g} / \mathrm{mL}$ in $0.4 \mathrm{~mL}$ of normal saline, as separate subcutaneous injections. These subjects were evaluated at 48 hours (for local reaction) and at 7 and 30 days after injection for evidence of an immune response.

Follow-up and monitoring. All subjects were evaluated 24 and 48 hours after each DC injection for the occurrence of DTH reaction or other early toxicity, and were seen in the clinic 7 and 30 days after each injection for measurement of immune responses. Subsequent follow-up was at 1-3 monthly intervals. In subjects who had preinjection skin tests, these tests were repeated at 30 days after completion of antigen-pulsed DC injections. Thirty days after all DC injections, and every 3 months thereafter, all subjects had a repeat hemogram, serum chemistry panel, and laboratory tests for rheumatoid factor and ANA. None of the patients had serologic evidence of intercur-

Table 2

DTH reaction to $T \mathrm{~T}$ : response to immunization

$$
\text { Before immunization After immunization }
$$

Erythema $(\mathrm{mm})$ Induration $(\mathrm{mm})$ Erythema $(\mathrm{mm})$ Induration $(\mathrm{mm})$

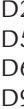

\begin{tabular}{cccc}
$2 \times 2$ & None & $20 \times 20$ & $8 \times 8$ \\
$8 \times 8$ & None & $40 \times 40$ & $10 \times 10$ \\
$9 \times 9$ & None & $28 \times 21$ & $17 \times 11$ \\
$7 \times 10$ & $5 \times 5$ & $22 \times 30$ & $10 \times 10$ \\
\hline
\end{tabular}

\section{Results} subjects are shown in Table 1 . Eight healthy subjects ( 5 HLA-A*0201-positive and 3 HLA-A*0201-negative) received an initial control injection of autologous DCs without antigen, followed a month later by antigenpulsed DC injection. In 1 additional subject (D5), the control injection before antigen-pulsed DCs was omitted. Pulsing of DCs with TT was omitted in 3 subjects who had significant induration and erythema $(>30 \mathrm{~mm})$ to a baseline TT skin test (D1, D3, D8). Four additional control subjects (3 HLA-A*0201-positive [A1-A3] and 1 HLA-A*0201-negative [A4]) were injected with antigen alone in the absence of adjuvant or DCs. The median cell dose of control DC injection was $2 \times 10^{6}$ cells; for antigen-pulsed DC injection, $2.8 \times 10^{6}$ cells. The percentage of injected $\mathrm{CD} 83^{+}$cells in the preparations was variable (range 19-95\%), owing to variable T-cell contamination; however, $>85 \%$ of the large cells were $\mathrm{CD}^{2} 3^{+}$.

Toxicities. Injection of the control DCs was well tolerated, without any local reaction or systemic toxicity (Table 1). A local reaction in the form of erythema without induration was seen in more than half of the subjects after antigen-pulsed DC injection. All antigen-pulsed DC injections were well tolerated, without systemic toxicity, with the exception of transient fatigue (D5) and low-grade fever (D6) on the day of the injection. Among the 4 subjects injected with antigens alone (without DCs), 3 developed erythema at the TT site (with induration in 1 subject), and 1 had a mild local reaction at the MP injection site, whereas no local reaction was observed at the KLH site in any of the subjects. None of the study participants experienced any rash or lymphadenopathy or developed any clinical evidence of autoimmunity or
Clinical characteristics. The clinical characteristics of the 
rheumatoid disease. One subject developed transient low-titer (1:40) ANA reactivity at 60 days after immunization, without any other serologic or clinical evidence of autoimmunity.

\section{Immune responses}

$\mathrm{CD}^{+} \mathrm{T}$-cell responses. There was no priming to $\mathrm{KLH}$, as assessed by antigen-specific proliferation after injections of the control DCs or antigen only at either the 7- or 30day time point (Figure 2a). In contrast, priming to KLH was seen in all 9 subjects after injection of antigen-pulsed DCs $(P<0.001)$. To illustrate the data, measurements on a representative subject (D1) are shown (Figure 2b). Priming to KLH was noted by 30 days after injection in 8 of 9 patients and by day 60 in all patients, but it was evident as early as 7 days after injection in 2 patients (not shown). In the 3 patients with longer follow-up, KLH priming was still detectable at 60-90 days after injection in 2 of 3 subjects. KLH-specific T-cell proliferative responses were mediated by $\mathrm{CD}^{+} \mathrm{T}$ cells, as depletion of $\mathrm{CD}^{+} \mathrm{T}$ cells did not alter the responses (Figure 2c).

Similarly, TT-specific immunity was boosted in 5 of 6 subjects, as assessed by antigen-specific proliferation $(P$ $=0.001$ ) (Figure 3 ). No boosting of TT responses was observed in the 3 subjects (D1, D3, D8) who did not receive TT-pulsed DCs. Of the 4 subjects who were skin tested to TT at baseline and 1 month after DC injection, all 4 (including D6, who failed to show an increase in proliferative response) demonstrated an enhanced DTH response to TT (Table 2 ).

$C D 8^{+} T$-cell responses in uncultured PBMCs. MP or influenza-specific IFN- $\gamma$-secreting cells were quantified in fresh PBMCs using an ELISPOT assay. There was no significant increase in MP or influenza-reactive IFN- $\gamma$ SFCs, after either the control DC injection or injection of antigen alone. In contrast, an enhancement of MP-specific T cells after antigen-pulsed DCs was seen in all $6 \mathrm{~A}^{*} 0201$-positive subjects $(P<0.001)$ and in none of the $3 \mathrm{~A}^{*} 0201$-negative control subjects (Figure 4a). To illustrate the data, measurements on a representative subject (D6) are shown (Figure 4b). Five of 6 subjects had $\geq 2$-fold increase in MP-reactive $T$ cells (median of 5-fold, corresponding to 100-200 MP-reactive $\mathrm{T}$ cells per $\left.10^{6} \mathrm{PBMCs}\right)$. Increases in influenza-specific $\mathrm{T}$ cells in the ELISPOT assay were seen in 4 of 6 subjects after immunization (D1, D2, D3, and D5; data not shown). Most MP-specific SFCs were $\mathrm{CD}^{+}$, as reactivity was lost by depletion of $\mathrm{CD}^{+}$, but not $\mathrm{CD}^{+}$, T cells (Figure 4c). When cryopreserved samples from before and after immunization were thawed together, similar findings were noted in the ELISPOT assay, but the number of SFCs was $40-50 \%$ less than in fresh PBMCs (not shown). The responding ELISPOT-positive cells were capable of responding to influenza virus as well as to MP. Analysis of lytic CD8 ${ }^{+}$cells in fresh bulk PBMCs failed to detect a significant increase in MP-specific CTLs in any of the subjects (not shown).

Recall $C D 8^{+} T$-cell responses in tissue culture. In contrast, a $\geq 2$-fold increase in MP-specific recall CTLs was seen in 5 of 6 HLA-A*0201-positive subjects and in none of the HLA-A*0201-negative control subjects (Figure 5a).

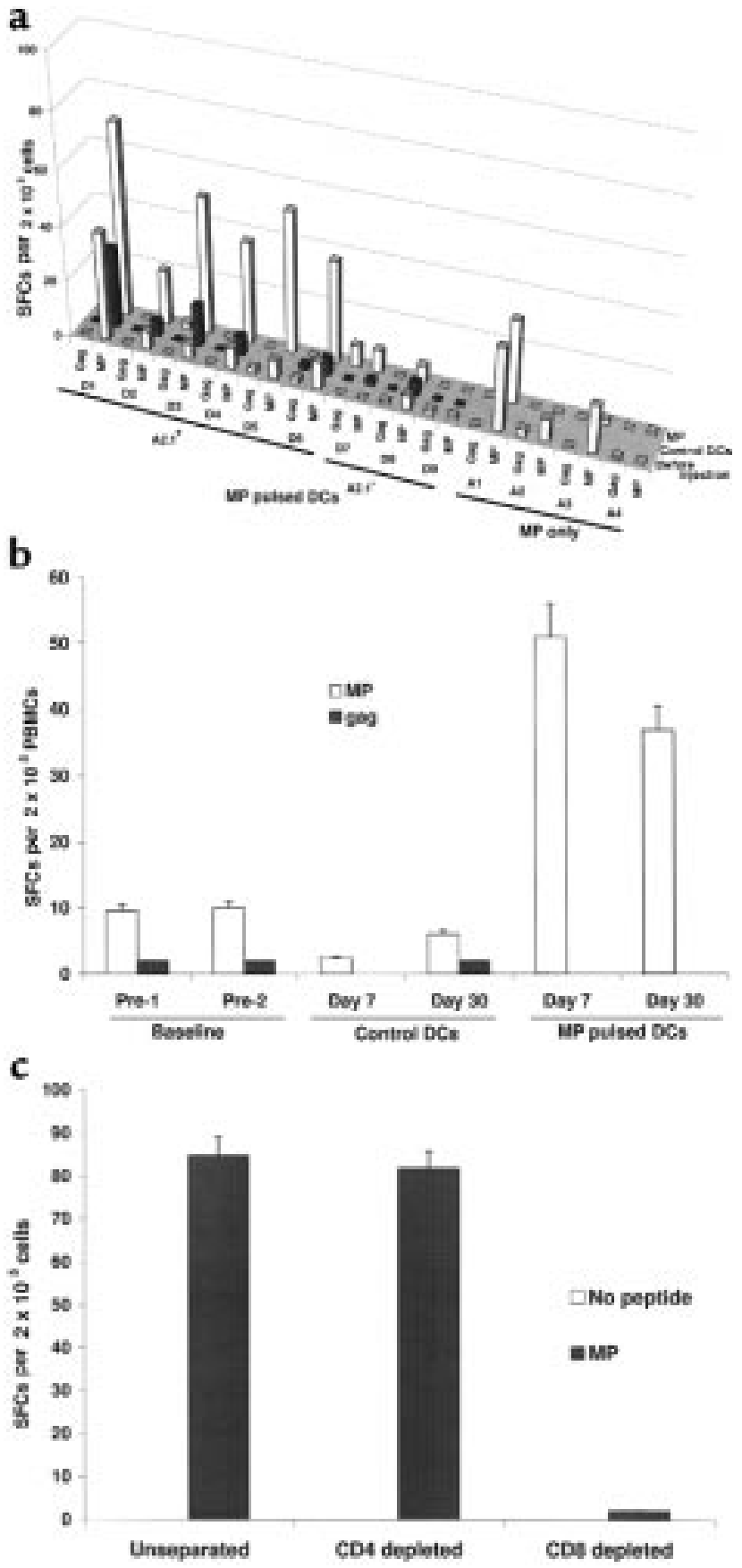

Figure 4

(a) Boosting of HLA-A*0201 restricted influenza MP-reactive T cells after MP-pulsed DCs. MP-reactive $T$ cells were quantified using an ELISPOT assay. HLA-A*0201 restricted gag peptide served as control. Results are shown as the number of SFCs per $2 \times 10^{5}$ PBMCs, at baseline and 30 days after the injection of control DCs, MP-pulsed DCs, or MP alone without DCs. SEM for all measurements was $<20 \%$. (b) Kinetics of boosting of MP-reactive T cells in a representative subject (D6). MP-reactive $T$ cells were quantified using an ELISPOT assay. HLA$A^{*} 0201$ restricted gag peptide served as control. For each assay, graded doses of PBMCs $\left(25 \times 10^{3}\right.$ to $2 \times 10^{5}$ cells per well $)$ were incubated with $10 \mu \mathrm{M}$ influenza MP. Results are shown as the number of SFCs per $2 \times 10^{5}$ PBMCs per well, at various time points. (c) $\mathrm{CD}^{+}$nature of the MP-reactive T cells in the ELISPOT assay. The presence of MP-reactive $T$ cells in unseparated PBMCs was compared with that after CD4 and CD8 depletion by panning. Data are from subject D1. 


\section{Figure 5}

(a) Boosting of MP-specific CTLs after MP-pulsed DCs. T cells were cocultured with DCs pulsed with $1 \mu \mathrm{M} \mathrm{MP}$ (unpulsed DCs as controls) for 7 days $(\mathrm{DC} / \mathrm{T}$ ratio $=30: 1)$, and lytic activity was tested against appropriate targets as described in Methods $(E / T$ ratio $=$ $20: 1)$. Data shown are from before injection and 30 days after control DC injection and antigen-pulsed DC injection, and represent percent MP-specific lysis after subtracting the background lysis with unpulsed DCs. SEM for all measurements was $<20 \%$. (b) Enhancement of MP-specific memory CTLs after MP-pulsed DC injection. T cells were cocultured with DCs pulsed with MP (unpulsed DCs as controls) for 7 days $(D C / T$ ratio $=1: 30)$, and lytic activity was tested against MP-pulsed or unpulsed T2 targets $(E / T$ ratio $=20: 1)$. Data shown represent percent MP-specific lysis after subtracting the background lysis with unpulsed DCs, in 2 representative subjects (D1 and D3), with longer follow-up. SEM for all measurements was $<10 \%$.

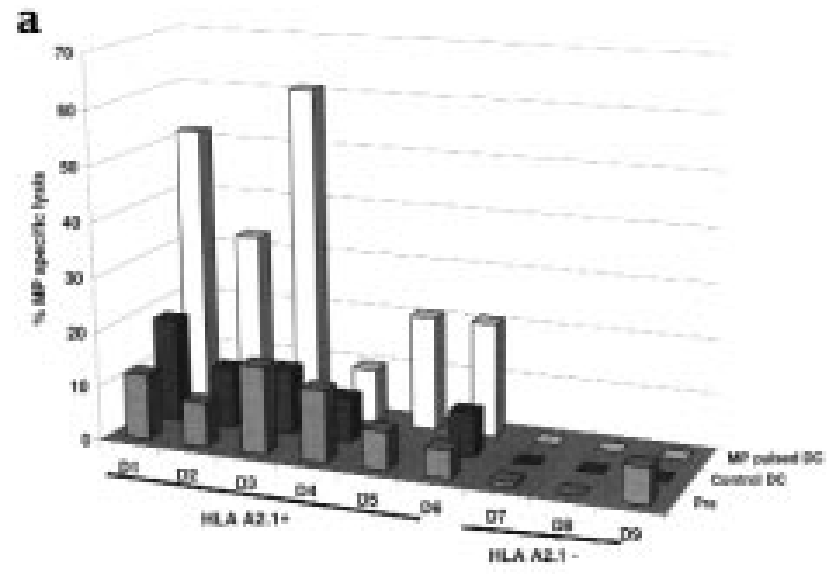

b
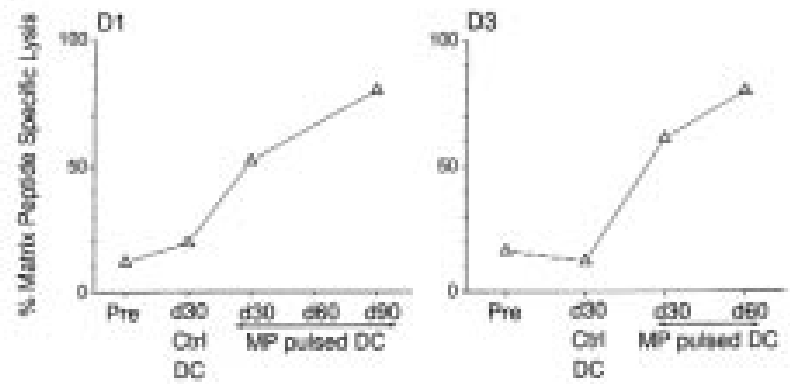

DC
In the 2 patients with a longer follow-up, the increased recall CTL responses to MP were maintained for up to 60-90 days after injection (Figure $5 \mathrm{~b}$ ).

When cryopreserved PBMCs from before and after immunization were thawed together and cocultured with MP-pulsed or unpulsed DCs, expansion of MP-specific $\mathrm{T}$ cells, assessed by tetramer binding, was noted in the available preimmunization samples from only 1 of the 3 subjects tested, which correlated with the subjects' preimmunization ELISPOT data (Figure 6). However, in the samples from after immunization with DCs, a significantly higher expansion of MP-specific $\mathrm{CD}^{+} \mathrm{T}$ cells was detected, with tetramer binding in all 3 samples assayed at 7 days (D5 and D6; Figure 6) and 90 days (D1; Figure 7) after immunization.

\section{Discussion}

Our study demonstrates that mature DCs are effective adjuvants in humans, capable of broadly expanding T-cell immunity - including not only CD4 ${ }^{+} \mathrm{T}$ helper cells, but also $\mathrm{CD}^{+}$effector and memory T cells. This is, to our knowledge, the first report using these DCs, and it provides the first controlled evidence of the immunogenicity of DCs in humans. Humans respond rapidly to a single injection of mature DCs, within a week in many cases, and within 30 days in all. The generated T-cell responses after a single injection are durable and last $>90$ days. In contrast, injection of the same antigens subcutaneously without DCs failed to generate or boost immunity. In studies that were carried out decades ago, injection of $\mathrm{KLH}$ at doses $>10 \mu \mathrm{g}$ was shown to generate T-cell immunity (24). However, the KLH preparations were not well defined, in particular for LPS contamination. In our study, when endotoxin-depleted KLH and TT were administered alone without any adjuvant at doses used to pulse DCs, the proteins failed to elicit measurable Tcell immunity, illustrating the value of DCs for both priming (in the case of KLH) and boosting (in the case of TT) CD4-positive T-cell responses. We did detect a weak antibody response to KLH in 3 of 4 subjects injected with KLH alone (M. Dhodapkar et al., unpublished data), indicating that a humoral, but not a T-cell, response was elicited in these patients. These data also add to the growing body of evidence that injection of DCs in humans is safe and clinically well tolerated.

The ability of DCs to enhance both antigen-specific $\mathrm{CD}^{+}$and $\mathrm{CD}^{+} \mathrm{T}$-cell responses is important, as both may be required for protective immunity against viruses and tumors and for the function of memory $\mathrm{CD}^{+} \mathrm{T}$ cells $(25,26)$. Previous approaches for eliciting $\mathrm{T}$-cell responses in humans have included the injection of class I restricted peptides (e.g., melanoma peptides) with noncellular adjuvants (27), recombinant viral vectors (e.g., poxviruses) (28), DNA vaccines (29), or immature DCs $(8,10)$. However, several injections have been required to generate detectable immunity. Furthermore, it has been necessary to use prolonged culture and repetitive in vitro stimulation with cytokines and antigen to detect these responses $(28,29)$. In direct contrast, we could detect the expansion of antigen-specific $\mathrm{CD}^{+} \mathrm{T}$ cells directly in freshly isolated T cells. We also showed an increase in T-cell function when the $\mathrm{T}$ cells from DC-immunized individuals were boosted in short-term (7-day) cultures. 


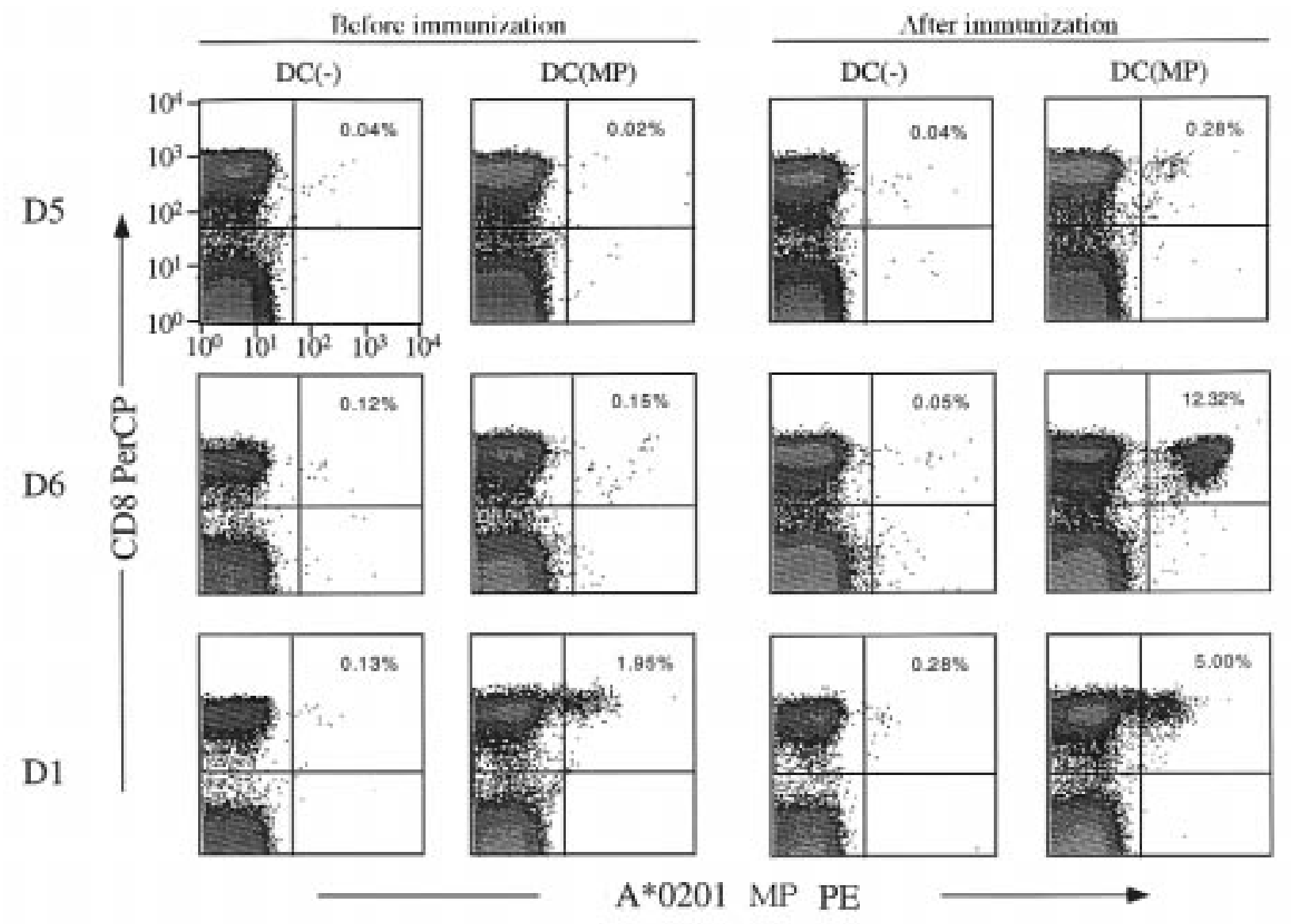

Figure 6

MP-specific memory CD8 ${ }^{+} T$ cells are expanded after MP-pulsed DC injection. PBMCs taken before or after immunization with MP-pulsed $D C$ s were thawed together and cultured with autologous unpulsed $[D C(-)]$ or MP-pulsed $[D C(M P)] D C s$. After a 7-day culture, the number of MP-specific CD8 ${ }^{+}$T cells was quantified by $A^{*}$ 0201-MP tetramer binding. (Top) D5, before and 7 days after immunization; (middle) D6, before and 7 days after immunization; (bottom) D1, before and 90 days after immunization. Percentage of tetramer-binding CD $8^{+} T$ cells is noted in the upper-right quadrant.

Specifically, there was a large increase in cells that bound MHC tetramers and killed targets. These findings illustrate the potency of DCs in generating T-cell immunity. A possible explanation for the more rapid, effective, and broad immunization that we observed may be our use of mature DCs, which are the most potent APCs in vitro (22), or maybe the subcutaneous route of administration, in contrast to other reports that have used immature DCs administered intravenously or intranodally $(8,10)$. However, direct comparison of various maturation stages of DCs and of DCs with other approaches is needed to evaluate their relative potency at eliciting $\mathrm{T}$-cell immunity.

A distinguishing feature of our study is the use of a complement of newer sensitive and quantitative assays (including ELISPOT and MHC tetramer-binding assay) to monitor induction of CD8 ${ }^{+} \mathrm{T}$-cell immunity $(15,16)$. These assays allow quantification of $\mathrm{CD}^{+} \mathrm{T}$ cells and their function (IFN- $\gamma$ production and cytolytic activity). The use of DCs as APCs in recall assays enables us to elicit information about T-cell memory without the need for in vitro cytokine stimulation, which is often employed in assays using other, less potent APCs. Together, the panel of $\mathrm{CD}^{+} \mathrm{T}$-cell assays indicates the capacity of DCs to stimulate effector and recall responses in humans.

$\mathrm{KLH}$ and TT were used in our subjects to monitor the stimulation of $\mathrm{CD} 4^{+} \mathrm{T}$ cells. These $\mathrm{CD} 4^{+} \mathrm{T}$ cells may also have provided help for the generation of $\mathrm{CD}^{+} \mathrm{T}$-cell responses, e.g., by "conditioning” DCs (30). Further clinical studies are needed to explore this possibility and to optimize variables such as DC maturational state, route of administration, dose, and schedule. Given the fact that tumors and viruses have several mechanisms to evade the immune response (25), it should be valuable to optimize the immune response, so that DCs can best be tested in the immunotherapy and prevention of viral and malignant disease in humans $(13,31)$.

\section{Acknowledgments}

This work was supported in part by a Clinical Research Career Development Award from the American Society of Clinical Oncology (to M.V. Dhodapkar), by grants from the National Institutes of Health (AI-40874 to R.M. Steinman; AI-39516 and AI-44628 to N. Bhardwaj; AI-44595 to D.F. Nixon; and M01-RR00102 to the General Clinical Research Center), and by the SLE Founda- 
tion (to N. Bhardwaj). We thank all subjects for their interest and participation in this study; Rockefeller University nursing staff for their help with patient care; Knut Wittkowski and Monnie Harper for guidance with statistical analysis; Lanie Fleischer for help with recruitment; Judy Adams for graphics; and Helen Bateman, Marie Larsson, and Anita Reddy for assistance during preclinical development.

1. Raychaudhuri, S., and Rock, K.L. 1998. Fully mobilizing host defense: building better vaccines. Nat. Biotechnol. 16:1025-1031.

2. Banchereau, J., and Steinman, R.M. 1998. Dendritic cells and the control of immunity. Nature. 392:245-252.

3. Cella, M., Sallusto, F., and Lanzavecchia, A. 1997. Origin, maturation and antigen presenting function of dendritic cells. Curr. Opin. Immunol. 9:10-16.

4. Celluzzi, C.M., Mayordomo, J.I., Storkus, W.J., Lotze, M.T., and Falo, L.D., Jr. 1996. Peptide-pulsed dendritic cells induce antigen-specific, CTL-mediated protective tumor immunity. J. Exp. Med. 183:283-287.

5. Mayordomo, J.I., et al. 1997. Bone marrow-derived dendritic cells serve as potent adjuvants for peptide-based antitumor vaccines. Stem Cells. 15:94-103.

6. Porgador, A., Snyder, D., and Gilboa, E. 1996. Induction of antitumor immunity using bone marrow-generated dendritic cells. J. Immunol. 156:2918-2926.

7. Zitvogel, L., et al. 1996. Therapy of murine tumors with tumor peptidepulsed dendritic cells: dependence on T cells, B7 costimulation, and T helper cell 1-associated cytokines. J. Exp. Med. 183:87-97.

8. Hsu, F.J., et al. 1996. Vaccination of patients with B-cell lymphoma using autologous antigen-pulsed dendritic cells. Nat. Med. 2:52-58.

9. Murphy, G., Tjoa, B., Ragde, H., Kenny, G., and Boynton, A. 1996. Phase I clinical trial: T-cell therapy for prostate cancer using autologous dendritic cells pulsed with HLA-A0201-specific peptides from prostate-specific membrane antigen. Prostate. 29:371-380.

10. Nestle, F.O., et al. 1998. Vaccination of melanoma patients with peptideor tumor lysate-pulsed dendritic cells. Nat. Med. 4:328-332.

11. Kundu, S.K., et al. 1998. A pilot clinical trial of HIV antigen-pulsed allogeneic and autologous dendritic cell therapy in HIV-infected patients. AIDS Res. Hum. Retroviruses. 14:551-560.

12. Wen, Y.J., Ling, M., Bailey-Wood, R., and Lim, S.H. 1998. Idiotypic protein-pulsed adherent peripheral blood mononuclear cell-derived dendritic cells prime immune system in multiple myeloma. Clin. Cancer Res. 4:957-962.

13. Schuler, G., and Steinman, R.M. 1997. Dendritic cells as adjuvants for immune-mediated resistance to tumors. J. Exp. Med. 186:1183-1187.

14. Bender, A., Sapp, M., Schuler, G., Steinman, R.M., and Bhardwaj, N. 1996. Improved methods for the generation of dendritic cells from non- proliferating progenitors in human blood. J. Immunol. Methods. 196:121-135

15. Altman, J.D., et al. 1996. Phenotypic analysis of antigen-specific T lymphocytes. Science. 274:94-96.

16. Lalvani, A., et al. 1997. Rapid effector function in $\mathrm{CD}^{+}$memory $\mathrm{T}$ cells. J. Exp. Med. 186:859-865.

17. Larsson, M., et al. 1999. A recombinant vaccinia virus based ELISPOT assay detects high frequencies of pol specific CD8+ T cells in chronically infected HIV-1 positive individuals. AIDS. 13:767-777.

18. Gotch, F., Rothbard, J., Howland, K., Townsend, A., and McMichael, A. 1987. Cytotoxic T lymphocytes recognize a fragment of influenza virus matrix protein in association with HLA-A2. Nature. 326:881-882.

19. Bender, A., Bui, L.K., Feldman, M.A., Larsson, M., and Bhardwaj, N. 1995. Inactivated influenza virus, when presented on dendritic cells, elicits human CD8+ cytolytic T cell responses. J. Exp. Med. 182:1663-1671.

20. Bhardwaj, N., et al. 1994. Influenza virus-infected dendritic cells stimulate strong proliferative and cytolytic responses from human CD8+ T cells. J. Clin. Invest. 94:797-807.

21. Dunbar, P.R., et al. 1998. Direct isolation, phenotyping and cloning of low-frequency antigen-specific cytotoxic $\mathrm{T}$ lymphocytes from peripheral blood. Curr. Biol. 8:413-416.

22. Reddy, A., Sapp, M., Feldman, M., Subklewe, M., and Bhardwaj, N. 1997. A monocyte conditioned medium is more effective than defined cytokines in mediating the terminal maturation of human dendritic cells. Blood. 90:3640-3646.

23. Zhou, L.-J., and Tedder, T.F. 1997. CD14+ blood monocytes can differentiate into functionally mature CD83+ dendritic cells. Proc. Natl. Acad. Sci. USA. 93:2588-2592.

24. Curtis, J.E., Hersh, E.M., Harris, J.E., McBride, C., and Freireich, E.J. 1970. The human primary immune response to keyhole limpet haemocyanin: interrelationships of delayed hypersensitivity, antibody response and in vitro blast transformation. Clin. Exp. Immunol. 6:473-491.

25. Pardoll, D.M. 1998. Cancer vaccines. Nat. Med. 4:525-531.

26. Zinkernagel, R.M., and Hengartner, H. 1997. Antiviral immunity. Immunol. Today. 18:258-260.

27. Rosenberg, S.A., et al. 1998. Immunologic and therapeutic evaluation of a synthetic peptide vaccine for the treatment of patients with metastatic melanoma. Nat. Med. 4:321-327.

28. Clements-Mann, M.L., et al. 1998. Immune responses to human immunodeficiency virus (HIV) type 1 induced by canarypox expressing HIV1MN gp120, HIV-1SF2 recombinant gp120, or both vaccines in seronegative adults. NIAID AIDS Vaccine Evaluation Group. J. Infect. Dis. 177:1230-1246

29. Wang, R., et al. 1998. Induction of antigen-specific cytotoxic T lymphocytes in humans by a malaria DNA vaccine. Science. 282:476-480.

30. Ridge, J.P., Di Rosa, F., and Matzinger, P. 1998. A conditioned dendritic cell can be a temporal bridge between a CD4+ T-helper and a T-killer cell. Nature. 393:474-478.

31. Steinman, R.M., and Germain, R.N. 1998. Antigen presentation and related immunological aspects of HIV-1 vaccines. AIDS. 12:S97-S112. 